\title{
Genetic immunization against cervical carcinoma: induction of cytotoxic T lymphocyte activity with a recombinant alphavirus vector expressing human papillomavirus type 16 E6 and E7
}

\author{
T Daemen, F Pries, L Bungener, M Kraak, J Regts and J Wilschut \\ Department of Medical Microbiology, Molecular Virology Section, University of Groningen, A Deusinglaan 1, 9713 AV Groningen, \\ The Netherlands
}

\begin{abstract}
Infection of genital epithelial cells with human papillomavirus (HPV) types 16 and 18 is closely associated with the development of cervical carcinoma. The transforming potential of these high-risk HPVs depends on the expression of the E6 and E7 early viral gene products. Since the expression of E6 and E7 is selectively maintained in premalignant and malignant cervical lesions these proteins are attractive candidates for immunotherapeutic and prophylactic strategies. This report describes the construction, characterization and the in vivo immunotherapeutic potential of recombinant Semliki Forest virus (SFV) expressing the HPV16 E6 and E7 proteins (SFV-E6E7). Western blot analysis and immunofluorescence staining demonstrated expression of E6 and E7 in BHK cells infected with SFV-E6E7. Immunization of mice
\end{abstract}

with SFV-E6E7 resulted in an efficient in vivo priming of HPV-specific CTL activity. The induced CTL lysed murine tumor cells transformed with the HPV16 genome and EL4 cells loaded with an immunodominant class I-binding HPV E7 peptide. CTLs could reproducably be induced by immunization with three injections of as few as $10^{5}$ infectious units of SFV-E6E7. Protection from tumor challenge was studied using the tumor cell line TC-1. Immunization with $5 \times 10^{6}$ SFV-E6E7 particles protected $40 \%$ of the mice from tumor challenge. These results indicate that E6E7 expression by the efficient and safe recombinant SFV system represents a promising strategy for immunotherapy or immunoprophylaxis of cervical carcinoma. Gene Therapy (2000) 7, 18591866.

Keywords: human papilloma virus; Semliki Forest virus vector; E6; E7; CTL

\section{Introduction}

From molecular, clinical and epidemiological studies it is evident that the high-risk human papilloma viruses HPV16 and HPV18 are linked to the development of precursor lesions of cervical cancer and invasive cervical carcinoma. ${ }^{1}$ The HPV genome encodes 7 early (E) nonstructural regulatory proteins, and two late (L) structural proteins. Integration of the viral DNA in the genome of the host cell, which is an essential step in HPV16- or HPV18-induced development of cervical carcinoma, results in a loss of E1- or E2-mediated transcriptional control. As a consequence the transformed cells overexpress the E6 and E7 proteins, initiating the malignant transformation process. ${ }^{2-6}$

Specific cell-mediated immunity is believed to play an essential role in the control of HPV infections and cervical carcinoma. This assumption is based on observations showing: (1) that HPV-induced lesions regress spontaneously in the majority of individuals, and (2) that immunodeficient patients develop significantly more HPV-related proliferative lesions in skin and anogenital tissue than immunocompetent individuals., ${ }^{7,8}$ In several animal models it has been demonstrated that the HPV E6 and E7 proteins, constitutively expressed in HPV-transformed cells, can act as targets for CTL-mediated tumor cell killing and stimulation of tumor-specific CTL activity. ${ }^{9-12}$

Induction of an antigen-specific CTL response requires intracellular processing of the target antigen and presentation of antigenic peptides by MHC class I molecules. This can be achieved efficiently with recombinant viral vectors. In the present study, our approach is to use the Semliki Forest virus (SFV) expression system. SFV belongs to the genus Alphavirus of the family of the Togaviridae. ${ }^{13}$ Alphaviruses consist of a nucleocapsid with one copy of a single-stranded RNA molecule surrounded by an envelope containing spike proteins. Alphavirus RNA has a positive polarity enabling the genomic RNA to initiate an infection when introduced into the cytoplasm of a cell. In addition, the RNA is self-replicating, since it encodes its own replicase, and replication results in highlevel expression of the viral proteins in host cells. A fulllength cDNA copy of the viral genome has been cloned in a bacterial plasmid including a prokaryotic DNAdependent RNA polymerase such that viral RNA can be transcribed in vitro. These RNA transcripts are fully infectious, ie introduction into cells suffices to initiate repli- 
cation and a full infection cycle, resulting in virus formation. ${ }^{14}$ Liljeström and coworkers developed a vector system that allows for efficient expression of foreign coding sequences as part of the SFV RNA replicon. ${ }^{14-16}$ A high biosafety level is obtained by separating the replicase and structural genes of the viral genome. Thus, recombinant virus particles can be produced that infect cells only once. In addition, the SFV helper (containing the structural genes) was mutated in the gene encoding one of the spike proteins. ${ }^{15}$ In effect, such virus particles cannot infect cells unless they are activated with exogenous protease.

In this article we describe the construction of recombinant SFV encoding HPV16 E6 and E7 and the cellular immune response in mice induced by these recombinant SFV-E6E7 particles.

\section{Results}

\section{Production and titer determination of SFV particles}

Recombinant SFV particles were produced in BHK cells by electroporation of recombinant and Helper 2 RNA into these cells. After $24 \mathrm{~h}$ the medium containing the virus was removed from the cells and the virus particles were purified. Titers were determined by immunofluorescence using an antibody against SFV-nsP3 (replicase). This antibody was chosen because replicase is present in all cells infected with recombinant SFV. Thus, titers of different recombinant SFVs can be determined, independent of the inserted foreign gene(s). Typically, titers of unpurified virus were $10^{9}-10^{10}$ infectious units $/ \mathrm{ml}$. After purification titers were between $10^{10}$ and $10^{11}$ infectious units $/ \mathrm{ml}$.

\section{Western blot analysis of E6 and E7 expression}

In order to verify that SFV-E6E7 induced expression of the recombinant E6 and E7 proteins, BHK cells were infected with SFV-E6E7 or SFV-LacZ serving as negative control. In Figure 1, Western blots of cell lysates probed with anti-HPV16 E6 (panel a) or anti-HPV16 E7 (panel b) are shown. Staining with the anti-E6 polyclonal antibody revealed a band with a $M_{\mathrm{r}}$ of approximately $17 \mathrm{kDa}$. Staining with the anti-E7 monoclonal antibody revealed a band with an apparent electrophoretic mobility of approximately $20 \mathrm{kDa}$. This $M_{\mathrm{r}}$ does not correspond to the calculated $M_{\mathrm{r}}(11 \mathrm{kDa})$ but is in agreement with other studies in which E7 was produced by eukaryotic as well as prokaryotic expression systems. ${ }^{17-20}$

\section{Expression of HPV16 E6 and E7 in SFV-E6E7 infected cells}

Expression of E6 and E7 was also analyzed by indirect immunofluorescence analysis of BHK cells infected with SFV-E6E7. A low particle-to-cell ratio was chosen such that not all cells in the wells would become infected, in order to visualize positive and negative cells within one microscopic field. As shown in Figure 2, a strong fluorescence of both E6 and E7 was found in infected cells. In general, a bright staining of E6 was found in the perinucleus and cytoplasm while E7 was mainly found in the perinucleus. Previous studies demonstrated localization of the HPV18 E6 protein in the nuclear matrix and in nonnuclear membranes ${ }^{21,22}$ and of HPV16 E7 in the nucleus. ${ }^{18}$ However, it is very likely that differences in staining pat-

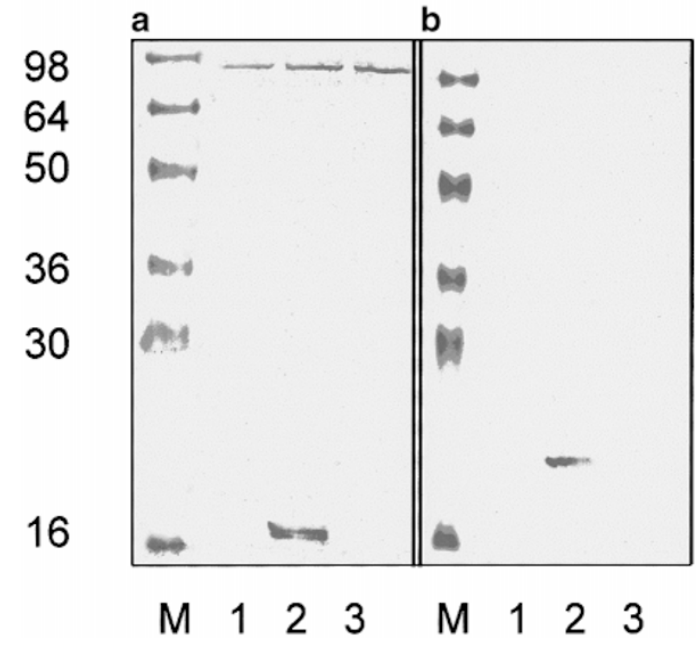

Figure 1 Western blot analysis of SFV-E6E7-transfected BHK cell extracts. BHK cells were infected with SFV-E6E7 particles or SFV-LacZ particles. After overnight incubation, the cellular proteins were extracted and analyzed by SDS-PAGE and immunoblotting. E6 was detected using a polyclonal rabbit-anti-HPV16 E6 antibody (a), E7 was detected using a monoclonal mouse-anti-HPV16 E7 antibody (b). Lanes 1: BHK21 cells not infected; lanes 2: BHK21 cells infected with SFV-E6E7 particles; lanes 3: BHK21 cells infected with SFV-LacZ particles; M: protein marker.

ern may be influenced by the amounts of proteins produced and the vector used for expression of the proteins. $^{18}$

\section{HPV-specific CTLs induced by immunization of mice with SFV-E6E7}

Mice were immunized s.c. and boosted twice (s.c. and i.p) with $10^{6}$ purified SFV-E6E7, SFV-LacZ particles or buffer, as a control. CTL activity was determined one week after the last booster immunization. After 11 and 18 days of in vitro restimulation the resulting effector cells were tested for their cytolytic activity against 13-2 target cells. As show in Figure 3, a strong CTL activity was induced upon administration of SFV-E6E7 particles (Figure $3 a$ and $b$, squares and diamonds), whereas no HPV-specific CTL activity was induced upon immunization with SFV-LacZ particles or PBS (Figure 3, triangles and crosses, respectively). The average level of cytolysis at day 11 (Figure 3a) increased slightly upon prolonged in vitro restimulation, ie 18 days culture (Figure $3 b$ ).

Since 13-2 cells only express the MHC class I H-2D ${ }^{\mathrm{b}}$ binding CTL epitope of HPV16 E7 peptide 49-57 (RAHYNIVTF), ${ }^{9}$ CTL clones directed against other epitopes on E6 and E7 are not detected. We also tested CTL activity against $\mathrm{C} 3$ cells as target cells, ie cells that express the entire HPV16 genome. CTLs present after 11 days of restimulation using C 3 cells as stimulator cells, lysed 132 cells (Figure $4 a$ ) and C3 cells (Figure $4 b$ ) to the same extent. This result suggests that the HPV16 E7 peptide 49-57 is one of the dominant CTL epitopes recognized by CTLs generated in C57BL/6 $\left(\mathrm{H}-2^{\mathrm{b}}\right)$ mice upon immunization with E6 and E7.

This suggestion is supported by the observation that target cells loaded with HPV16 E7 49-57 were recognized and lysed to a very high level. Figure 5a shows CTL activity, induced in two mice immunized with $10^{6} \mathrm{SFV}$ E6E7 particles, against EL4 cells loaded with the E7 49- 

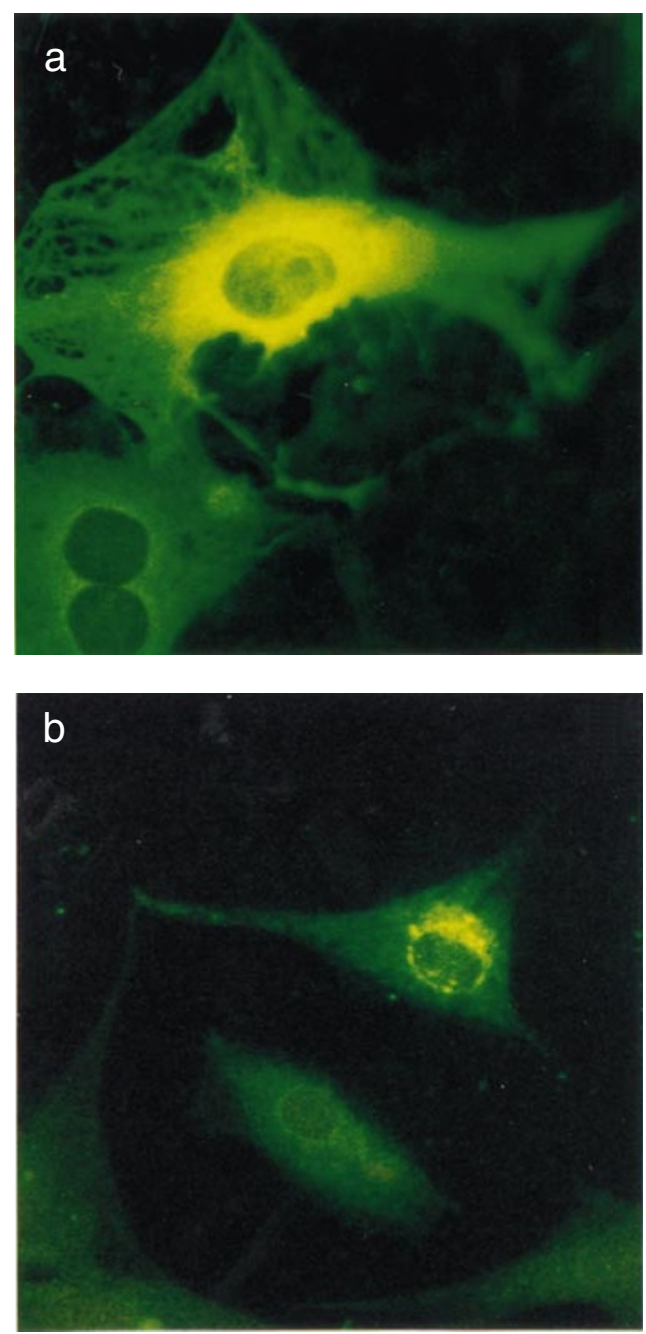

Figure 2 Intracellular localization of E7 in SFV-E6E7 infected BHK cells BHK21 cells were infected with SFV-E6E7. After overnight incubation, the cells were stained using anti-HPV16 E6 or anti-HPV16 E7 antibodies. (a) Immunofluorescent staining of SFV-E6E7 infected cells with antiHPV16 E6; (b) immunofluorescent staining of SFV-E6E7 infected cells with anti-HPV16 E7. Original magnification $\times 40$.

57 peptide as targets. On the other hand, mice immunized with SFV-LacZ particles or PBS did not recognize peptide-loaded EL4 cells (Figure 5a, triangles and crosses, respectively). In addition, unloaded EL4 cells were not recognized and lysed by these CTLs (Figure 5b).

To determine the minimal effective dose of SFV-E6E7 particles, mice were immunized and boosted twice with $10^{4}, 10^{5}$ or $10^{6}$ particles per immunization. In Figure $6 \mathrm{a}$ and $b$, the results of two separate experiments, each including two mice per injection dose, are given. In both experiments, immunization with $10^{6}$ SFV-E6E7 particles (squares) but also with as few as $10^{5}$ particles (diamonds), resulted in all mice developing an HPV-specific CTL response. Immunization with $10^{4}$ particles (Figure 6, solid triangles), resulted in a low but detectable response in two of four mice.

\section{Antitumor responses induced by immunization of mice with SFV-E6E7 particles}

To examine whether recombinant SFV particles could generate protective immunity against a subsequent
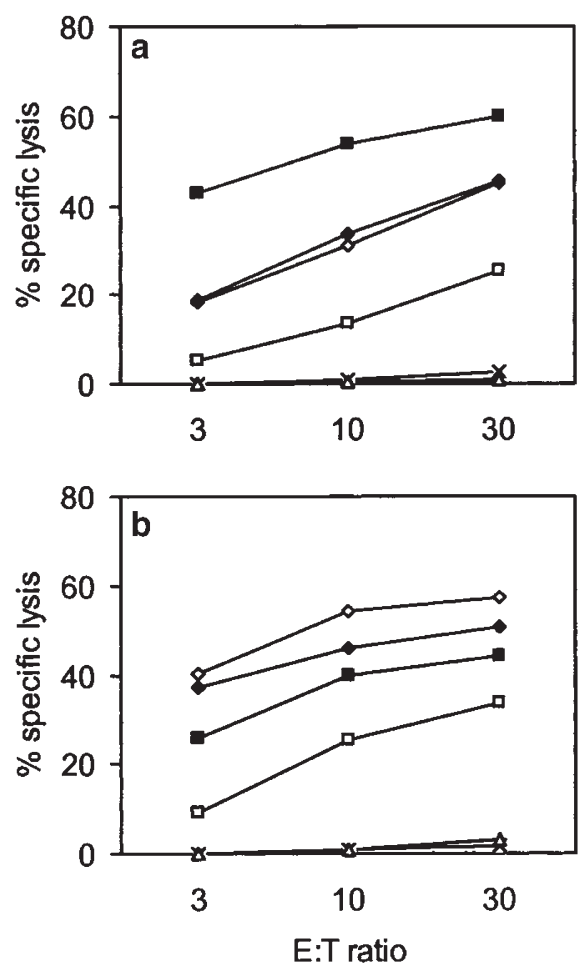

Figure 3 CTL activity induced upon immunization with SFV-E6E7 particles, as determined after an 11 and 18 day in vitro restimulation. Mice were immunized s.c. and boosted twice (s.c. and i.p) with purified $10^{6}$ SFV-E6E7 ( $n=4$, open and closed squares and diamonds), SFV-LacZ particles (triangles) or PBS (crosses), as a control. CTL activity was determined 1 week after the last booster immunization. After 11 days (a) and 18 days $(b)$ in vitro restimulation the resulting effector cells were tested for cytolytic activity against 13-2 target cells in triplicate well assay. Shown are the levels of cytolysis at different effector to target ratios. The standard errors of the means of the triplicate determinations were always $<10 \%$ of the value of the mean.

tumor challenge, mice were immunized and boosted with SFV-E6E7 particles and challenged s.c. with TC-1 cells, tumor cells expressing HPV16 E6E7. Tumor inoculation studies performed before initiating these immunization studies revealed that s.c. inoculation of $2 \times 10^{4} \mathrm{TC}-1$ cells reproducably induced tumors within 2 to 4 weeks after inoculation in all mice tested $(n=15)$. Figures 7 and 8 show the combined results of two separate immunization studies. Control mice, injected with PBS $(n=10)$ or SFVLacZ particles $(n=10)$ developed palpable tumors within 2 to 4 weeks after tumor cell inoculation (Figure 7a and b, respectively; Figure 8, open circles and open squares, respectively). Immunization with $10^{6} \mathrm{SFV}-\mathrm{E} 6 \mathrm{E} 7 \mathrm{particles}$ $(n=10)$ resulted in a delay in tumor onset in $50 \%$ of the mice as compared with control mice, with one of 10 mice not developing a tumor (Figure 7c; Figure 8, diamonds). Upon immunization with a five-fold higher dose of SFVE6E7 particles two of five mice did not develop a tumor (Figure 7d; Figure 8, closed squares).

\section{Discussion}

This report describes the construction, characterization and cellular immunotherapeutic potential of recombinant SFV particles encoding the early proteins E6 and E7 of HPV16. The ultimate aim of our studies is to develop an 

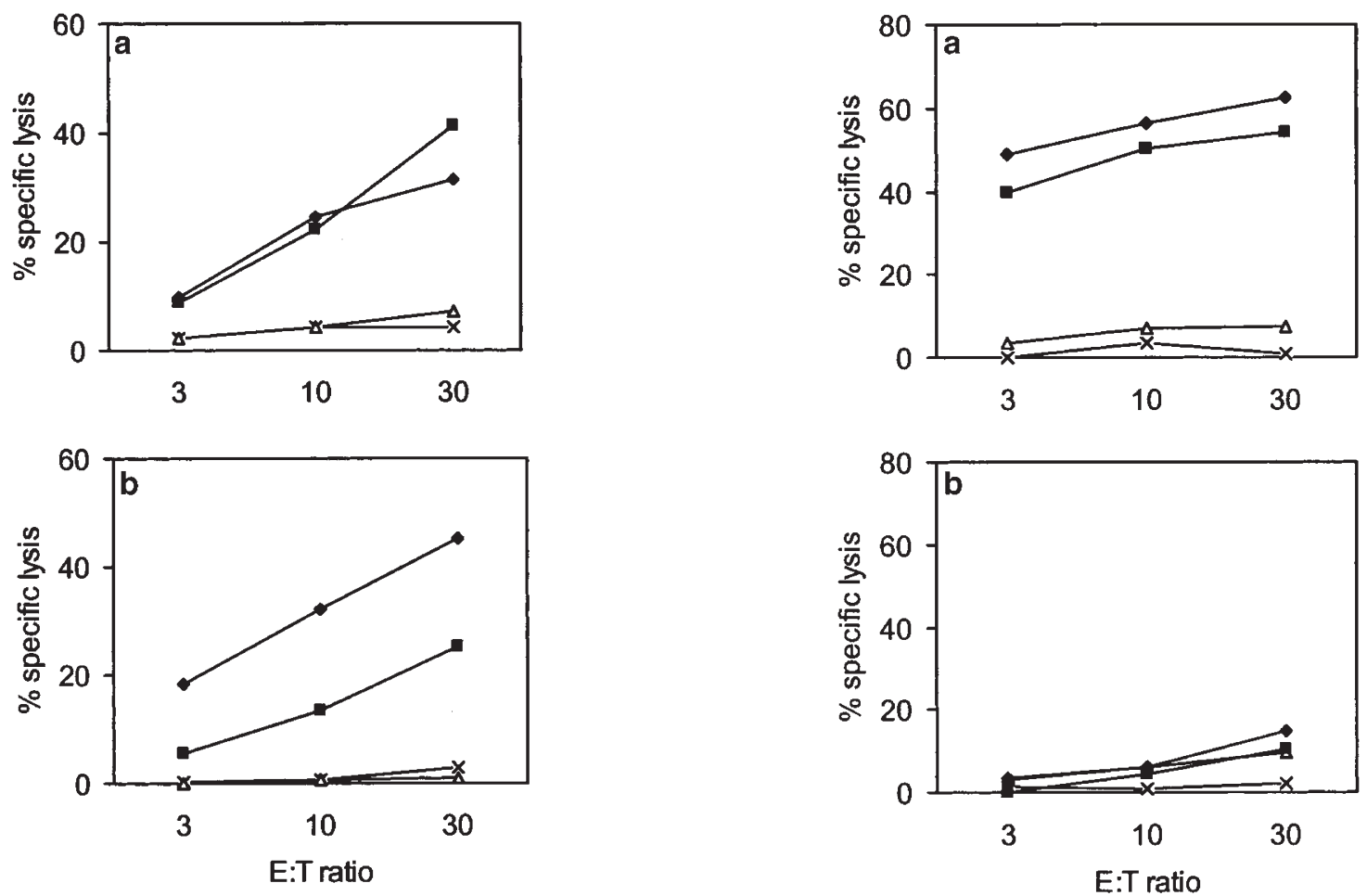

Figure 4 Recognition and lysis of HPV16-transformed C3 cells as well as 13-2 cells expressing the H-2Db-binding HPV16 CTL epitope by CTLs induced upon immunization with SFV-E6E7 particles. Mice were immunized s.c. and boosted twice (s.c. and i.p) with purified $10^{6}$ SFV-E6E7 ( $n=2$, squares and diamonds), SFV-LacZ particles (open triangles) or PBS (crosses), as a control. After 11 days in vitro restimulation the resulting effector cells were tested for cytolytic activity against 13-2 target cells (a) and C3 cells (b) in triplicate well assay. Shown are the levels of cytolysis at different effector to target ratios. The standard errors of the means of the triplicate determinations were always $<10 \%$ of the value of the mean.

effective immunization strategy for the treatment and/or prevention of HPV-induced cervical carcinoma.

Immunization of mice with SFV particles encoding HPV16 E6 and E7 resulted in a HPV-specific CTL response. Three injections of as few as $10^{4} \mathrm{SFV}$ particles sufficed for the induction of a CTL response in $50 \%$ of the mice, while three immunizations with $10^{5} \mathrm{SFV}$ particles induced a HPV-specific CTL response in all mice tested. Increasing the dose to $10^{6} \mathrm{SFV}$ particles per injection resulted in a reproducible CTL response with a high level of specific tumor cell lysis. In vitro blocking experiments with antibodies against CD4 and CD8 revealed that the lytic activity was due to $\mathrm{CD} 8^{+} \mathrm{T}$ cells; no inhibition was found with anti-CD4 antibodies (not shown). Tumor challenge experiments demonstrated that immunization with $10^{6} \mathrm{SFV}$-E6E7 particles resulted in a delay in tumor onset while one of 10 mice did not develop a tumor. Upon immunization with a five-fold higher dose of SFVE6E7 particles $40 \%$ of the mice did not develop a tumor.

In the last few years a number of peptide/proteinbased or genetic immunization strategies have been described for the induction of HPV-specific CTL activity. ${ }^{10-12}$ Major drawbacks associated with a peptidebased approach include the problem of MHC polymorphism and the risk of inducing $\mathrm{T}$ cell tolerance rather than $\mathrm{T}$ cell activation. Due to the induction of specific $\mathrm{T}$ cell tolerance, vaccination with a tumor-specific peptide

has been shown to result in an enhanced outgrowth of the tumor. ${ }^{23}$ Immunization with larger proteins would overcome these problems, but requires efficient antigen delivery systems and/or safe adjuvants for efficient immune priming. Several groups have described the induction of HPV-specific CTL responses in mice upon immunization with recombinant vaccinia virus expressing HPV E6 or $\mathrm{E}^{24,25}$ or with syngeneic cells retrovirally transfected with the HPV E6 gene. ${ }^{26}$ In a phase I/II trial involving eight patients with late stage cervical cancer, vaccination with recombinant vaccinia virus expressing HPV18 E6 and E7 induced HPV-specific CTLs in one of three evaluable patients. ${ }^{27}$ Potential drawbacks associated with the use of viral vector systems are immune responses against viral proteins in pre-immune patients (vaccinia virus) or integration of recombinant genes into the host cell genome (retrovirus). Especially, when the recombinant virus encodes oncoproteins such as HPV E6 or E7, the risk of integration into the host cell genome is a point of major concern.

We have chosen the SFV expression system, which apart from its transfection efficiency and high biosafety, would appear to be especially suited to induce cellular immune responses against oncoproteins such as HPV16 E6 and E7 safely. First, SFV is an RNA virus replicating in the cell cytosol; therefore, there is no risk of integration of the E6 and E7 genes in the cellular genome. Moreover, 

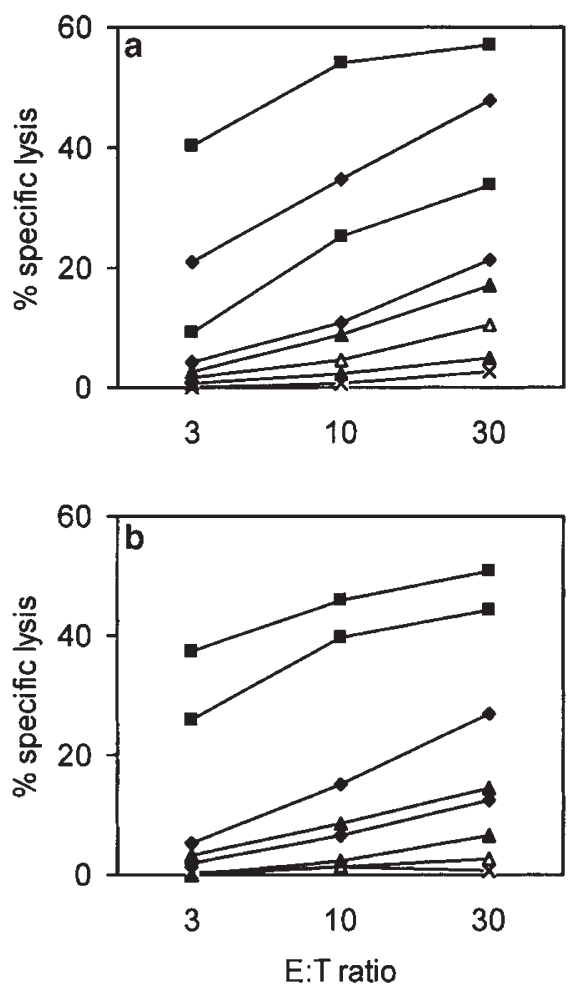

Figure 6 CTL activity in mice immunized with various doses of SFVE6E7 particles. In two separate experiments, mice were immunized s.c. and boosted twice (s.c. and i.p) with purified $10^{4}$ (solid triangles), $10^{5}$ (solid diamonds) or $10^{6}$ (solid squares) SFV-E6E7 particles, $10^{6} \mathrm{SFV}$ LacZ particles (open triangles) or PBS (crosses), as a control. CTL activity was determined 1 week after the last booster immunization. After 18 days in vitro restimulation the resulting effector cells were tested for cytolytic activity against 13-2 cells in triplicate well assay. Shown are the levels of cytolysis at different effector to target ratios of two individual experiments. The standard errors of the means of the triplicate determinations were always $<10 \%$ of the value of the mean.

SFV infection is cytolytic by apoptosis. ${ }^{13,28}$ Therefore, no genetic information of E6 and E7 will persist for more than 1 week after injection. In addition, no other vector proteins are produced, other than small amounts of viral replicase. Berglund et $a l^{29}$ demonstrated that immune responses against the vector itself did not inhibit boost responses by subsequent immunizations with the same vector.

Recognition by the immune system of virally infected cells or tumor cells occurs via virus- or tumor-specific antigenic peptides presented in the context of MHC class
I molecules. Infection of cells with recombinant SFV particles results in the production of the recombinant protein within the cytoplasm permitting presentation of the recombinant protein via the conventional MHC class I presentation route. However, for the induction of tumoror virus-specific CTLs, antigen presentation has to be accompanied by costimulatory signalling. Costimulatory molecules are confined to professional antigen-presenting cells (APCs). Therefore, the CTL response induced upon immunization with SFV-E6E7 particles may occur through transfection of APCs in vivo. Alternatively, APCs may take up residues of other cells that have been transfected in a process of cross-priming. The uptake of debris from infected cells by APCs is expected to be very efficient since an SFV infection induces apoptotic cell death. ${ }^{13,28}$ Upon uptake of infected-cell material (exogenous antigen) the recombinant protein will be processed and presented by MHC class II molecules thereby activating $\mathrm{CD} 4^{+} \mathrm{T}$ helper cells. In addition, dendritic cells and macrophages are able to present exogenous antigen in the context of MHC class I molecules for presentation to and activation of $\mathrm{CD}^{+} \mathrm{T}$ cells. ${ }^{30}$ Thus, both arms of the cellular immune system, essential for the induction of an optimal immune response will be activated upon administration of recombinant SFV particles, thereby eliciting a potent CTL response. Moreover, SFV immunization will introduce both class I and class II antigenic epitopes into one and the same the APC which has recently been demonstrated to be required for a full activation of APCs. ${ }^{31-34}$ As demonstrated by Zhou et al $l^{35}$ immunization of mice with SFV particles encoding for the nucleoprotein of influenza virus not only induces influenza-specific CTL activity, but also an nucleoproteinspecific antibody response. This observation supports the hypothesis of cross-priming and indirect presentation of antigenic peptides.

In conclusion, we have demonstrated that immunization of mice with recombinant SFV-E6E7 particles induces a potent CTL response against HPV-transformed tumor cells. This promising result, combined with studies showing the high efficacy of the SFV system for priming the immune system of mice as well as primates, $29,35,36$ and the recent development of the extremely safe two-helper system $^{37}$ provide the essential steps towards the design of an effective immunization strategy for the treatment and prophylaxis of HPV-induced cervical carcinoma.
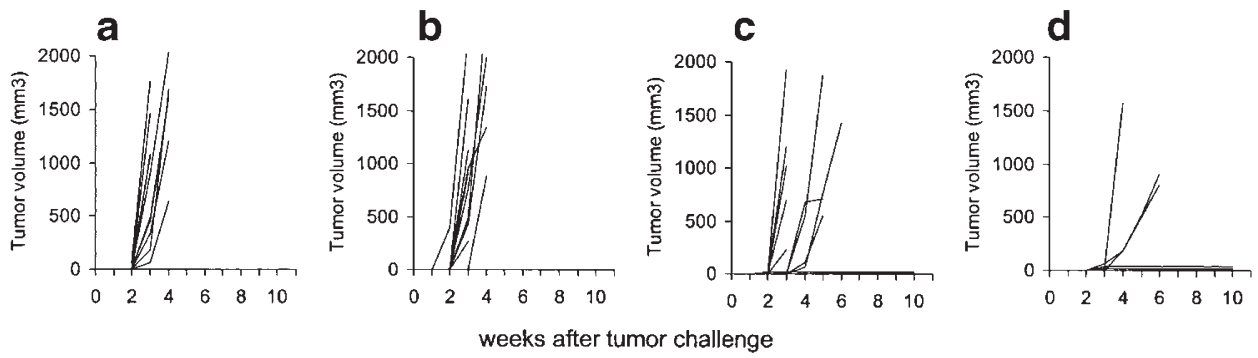

Figure 7 Growth of TC-1 tumor cells in SFV-E6E7 immunized mice. Mice were immunized s.c. and boosted twice (s.c. and i.p.) with PBS (a; $n=10)$, $5 \times 10^{6} \mathrm{SFV}$-LacZ particles $(b ; n=10), 10^{6} \mathrm{SFV}$-E6E7 particles $(c ; n=10)$ or $5 \times 10^{6}$ SFV-E6E7 particles $(d ; n=5)$. Tumor growth was monitored twice weekly. Each line represents the tumor volume of a separate mouse. 


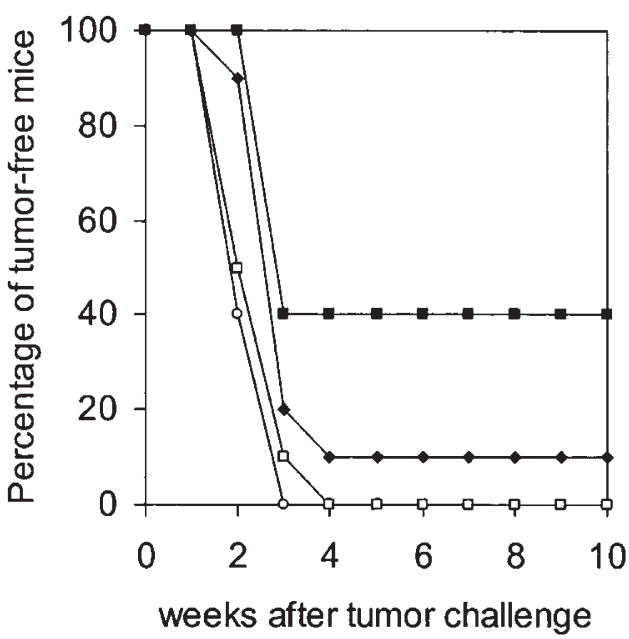

Figure 8 Growth of TC-1 tumor cells in SFV-E6E7 immunized mice. Mice were immunized s.c. and boosted twice (s.c. and i.p.) with PBS ( $n=10$; open circles), $5 \times 10^{6}$ SFV-LacZ particles ( $n=10$; open squares), $10^{6}$ SFV-E6E7 particles $\left(n=10\right.$; solid diamonds) or $5 \times 10^{6}$ SFV-E6E7 particles ( $n=5$; solid squares). Tumor growth was monitored twice weekly. Shown are the percentages of mice with non-palpable tumors.

\section{Materials and methods}

\section{Cell lines}

Baby hamster kidney cells (BHK-21) were obtained from the American Type Culture Collection (CCL-10). The cells were grown in GMEM (Life Technologies, Paisley, UK) containing 5\% fetal calf serum (PAA Laboratories, Linz, Austria). C3 cells, 13-2 cells and TC-1 cells were kindly provided by Dr C Melief and Dr R Offringa (Leiden University, The Netherlands). The C3 cell line was derived from C57BL/6 (H-2 ${ }^{\mathrm{b}}$ ) embryonic cells transfected with a plasmid containing the complete HPV16 genome. The 132 cell line was generated from $\mathrm{C} 57 \mathrm{Bl} / 6\left(\mathrm{H}-2^{\mathrm{b}}\right)$ embryonic cells transfected with the E1 region of adenovirus type 5 in which the adenoviral E1A epitope SGPSNTPPEI is replaced by a HPV16 E7 CTL epitope, AA 49-57 (RAHYNIVTF) (R Offringa, personal communication). The TC-1 cell line was generated from $\mathrm{C} 57 \mathrm{Bl} / 6$ primary lung epithelial cells with a retroviral vector expressing HPV16 E6E7 plus a retrovirus expressing activated c-Haras. ${ }^{25}$ EL4 cells were kindly provided by Dr L Leserman (Centre d'Immunologie de Marseille-Luminy, France). C3, 13-2, TC-1 and EL4 cells were grown in IMDM (Life Technologies) supplemented with $10 \%$ fetal calf serum. Both media contained penicillin and streptomycin (Life Technologies; $100 \mathrm{U} / \mathrm{ml}$ and $100 \mu \mathrm{g} / \mathrm{ml}$, respectively).

\section{Mice}

Specific pathogen-free female C57Bl/6 mice (Harlan CPB, Zeist, The Netherlands) were between 6 and 10 weeks of age at the start of the immunization protocols.

\section{Peptide}

The HPV16 $\mathrm{H}-2 \mathrm{D}^{\mathrm{b}}$ binding E7 peptide RAHYNIVTF (residue 49-57) was synthesized and purified by Dr JW Drijfhout (Academic Hospital Leiden, The Netherlands). The peptide was analyzed by reverse phase HPLC and found to be over $90 \%$ pure.

\section{Cloning of HPV16 E6 and E7 in pSFV3}

pSFV-Helper $2^{16}$ and pSFV3 ${ }^{15}$ were kindly provided by Dr P Liljeström (Karolinska Institute, Stockholm, Sweden). The HPV16 E6 and E7 genes were obtained from the plasmid pRSV-HPV16E6E7,38 which was kindly provided by Dr J Ter Schegget (Free University, Amsterdam, The Netherlands). In this plasmid the HPV16 E6 and E7 genes are present in tandem, with a stop codon after the E6 gene. Amplification of the E6E7 tandem gene was done by PCR using the following primers, written in 5 to 3 direction: GACGGATCCAAAGAGAACTCC AAT G (E6 forward) and GAGAATTCGGATCCGC CATGGTAGATTAT (E7 reverse). The PCR product was digested with BamHI and cloned into the BamHI site of pGEM7Zf + . After sequence confirmation, the E6E7 fragment was cloned into the unique BamHI site of pSFV3, producing pSFV3-E6E7.

Production and purification of recombinant SFV particles pSFV3-LacZ ${ }^{15}$ was a kind gift from Dr P Liljeström. The pSFV3-E6E7, pSFV3-LacZ and the pSFV-Helper 2 plasmids were isolated using the Qiagen midi plasmid purification kit and linearized by digestion with SpeI (Life Technologies). RNA was synthesized from the linearized DNA by in vitro transcription using SP6 RNA polymerase (Amersham Pharmacia Biotech, Piscataway, NJ, USA). Capping analogue was obtained from Life Technologies. Fifteen $\mu \mathrm{g}$ SFV3-E6E7 or SFV3-LacZ and $7.5 \mu \mathrm{g}$ SFVHelper 2 RNA were admixed and cotransfected into $8 \times 10^{6} \mathrm{BHK}$ cells in $0.8 \mathrm{ml}$ GMEM by electroporation using the Biorad Gene Pulser II (two pulses of 850 $\mathrm{V} / 25 \mu \mathrm{F}$; Biorad, Hercules, CA, USA). After pulsing, the cells were suspended in $10 \mathrm{ml}$ GMEM and cultured for $36 \mathrm{~h}$ at $37^{\circ} \mathrm{C}$ and $5 \% \mathrm{CO}_{2}$. The medium, containing the SFV-E6E7 or SFV-LacZ particles was centrifuged twice in a JA 20 rotor (Beckman, St Paul, MN, USA) at 1800 r.p.m. (ie $40000 \mathrm{~g}$ at $r_{\max }$ ) to remove cells and cellular debris.

The SFV particles were purified on a discontinous sucrose density gradient ( $2 \mathrm{ml}$ of a $15 \%$ sucrose solution $(\mathrm{w} / \mathrm{v})$ and $1 \mathrm{ml}$ of a $50 \%$ sucrose solution $(\mathrm{w} / \mathrm{v})$ in TNEbuffer (50 mm Tris-Cl, $100 \mathrm{~mm} \mathrm{NaCl}, 1 \mathrm{~mm}$ EDTA, pH 7.4)). Virus was collected from the interface. Sucrose was removed from the virus solution by overnight dialysis against TNE-buffer. The virus suspension was concentrated approximately 10-fold (Centricon 30 filter; Millipore, Bedford, MA, USA), quickly frozen in $\mathrm{N}_{2}$ and stored in aliquots at $-80^{\circ} \mathrm{C}$.

Before use, SFV particles were incubated with 1/20 volume of $\alpha$-chymotrypsin ( $10 \mathrm{mg} / \mathrm{ml}$; Sigma Chemical, St Louis, MO, USA) for $30 \mathrm{~min}$ at room temperature to cleave the mutated spike proteins. Subsequently, $\alpha$-chymotrypsin was inactivated by the addition of 0.5 volume of aprotinin ( $2 \mathrm{mg} / \mathrm{ml}$; Sigma Chemical).

\section{Titer determination of SFV particles}

Recombinant SFV particles were titrated by serial dilution on monolayers of BHK cells. After infection and overnight incubation the cells were fixed for $10 \mathrm{~min}$ in $10 \%$ acetone and stained using a polyclonal rabbit antireplicase (nsP3) antibody (a kind gift from Dr $\mathrm{T}$ Ahola, Biocentre Viiki, Helsinki, Finland) as primary antibody and FITC-labeled goat-anti-rabbit IgG as a secondary antibody (Southern Biotech. Ass., Birmingham, AL, USA). Positive cells were counted and the titer was determined after correcting for the dilution factor and the 
dilution caused by the activation and the volume of particles added.

\section{Analysis of E6 and E7 expression by Western blotting} BHK cells were infected with SFV-E6E7 particles or as a control with SFV-LacZ particles. After overnight incubation, the cells were harvested and lysed in lysis buffer (50 mm Tris.Cl, $5 \mathrm{~mm}$ EDTA, $150 \mathrm{~mm} \mathrm{NaCl}$, 0.5\% Triton X-100, pH 7.4). Cell-free extracts were analyzed by SDSPAGE. The proteins were blotted on to PVDF membrane (Immobilon-P; Millipore Corp, Bedford, MA, USA) and E6 and E7 were detected with a polyclonal rabbit-antiHPV16 E6 antibody (a kind gift from Dr I Jochmus, Deutsches Krebsforschungszentrum, Heidelberg, Germany) and a monoclonal mouse-anti-HPV16 E7 antibody (Zymed, South San Francisco, CA, USA), respectively. After incubation with alkaline phosphatase-linked secondary antibodies the blots were stained with nitroblue tetrazolium and 5-bromo-4-chloro-3-indolylphosphate (Sigma Chemical).

\section{Indirect immunofluorescence analysis of E6 and E7 in SFV-E6E7 infected cells}

In an eight-well culture chamber slide (Life Technologies) a monolayer of BHK cells was infected with SFV-E6E7. Fixation of the cells and staining was done as described for the immunofluorescence with anti-replicase, except for the used antibodies. As primary antibodies, antiHPV16 E6 or anti-HPV16 E7, as mentioned above, were used. The secondary antibodies were FITC-labeled antirabbit IgG and anti-mouse IgG, respectively (Southern Biotech. Ass.).

\section{Immunizations}

Mice were immunized subcutaneously (s.c. and intraperitoneally (i.p.)) and boosted twice with a 2-week interval, with $10^{4}$ to $5 \times 10^{6} \mathrm{SFV}$-E6E7 particles. As negative controls, mice were injected with equal doses of SFV-LacZ particles or PBS.

\section{CTL assay}

Seven to 21 days after the last booster immunization, spleen cells were isolated and cocultured with irradiated (100 Gy) C3 cells in a ratio of $25: 1$, in $25 \mathrm{~cm}^{2}$ culture flasks, placed upright. After 1 and 2 weeks in culture, cells were harvested and restimulated with irradiated naive spleen cells $(30 \mathrm{~Gy})$ and irradiated C3 cells in a ratio of 2:5:0.1 in 24-well plates in the presence of $4 \mathrm{IU}$ of recombinant hIL2/ml (Strathmann Biotech, Hamburg, Germany). Five days after the first and/or second restimulation, cells were harvested and a CTL assay was performed by a standard $4 \mathrm{~h}{ }^{51} \mathrm{Cr}$ release assay in triplicate determinations. Target cells were labeled for $1 \mathrm{~h}$ with 3.7 MBq ${ }^{51} \mathrm{Cr} / 10^{6}$ cells in $100 \mu \mathrm{l}$ medium $\left({ }^{51} \mathrm{Cr}\right.$ was from Amersham, London, UK). EL4 target cells were loaded with the HPV16 E7 49-57 (RAHYNIVTF) peptide by a $1 \mathrm{~h}$ incubation of the cells in the presence of $15 \mu \mathrm{g} / \mathrm{ml}$ of peptide in $100 \mu \mathrm{l}$ of culture medium before labeling the cells with ${ }^{51} \mathrm{Cr}$. The mean percentage of specific ${ }^{51} \mathrm{Cr}$ release of triplicate wells was calculated according to the formula: $\%$ specific release $=[$ experimental release spontaneous release)/(maximal release-spontaneous release)] c.p.m. $\times 100$. The spontaneous ${ }^{51} \mathrm{Cr}$ release was always $<15 \%$. The standard errors of the means of the triplicate determinations were $<10 \%$ of the value of the mean.

\section{Tumor challenge experiments}

Mice were immunized and boosted as described above with $10^{6}$ to $5 \times 10^{6}$ SFV-E6E7 particles, SFV-LacZ particles or PBS. One week after the last booster immunization the mice were challenged s.c. with $2 \times 10^{4}$ TC- 1 cells suspended in $0.2 \mathrm{ml}$ Hank's buffered salt solution (Life Technologies). Tumor measurements were always done by the same skilled technician. At a tumor volume of approximately $1000 \mathrm{~mm}^{3}$, the mice were killed.

\section{Acknowledgements}

We thank Dr P Liljeström and Dr C Melief and their coworkers for advice and stimulating discussions and $\mathrm{B}$ Dontje and J Wijbenga for expert technical assistance. Part of this work was supported by Inex Pharmaceuticals Corporation (Burnaby, BC, Canada) and the EU Biotechnology and Biomedicine and Health Programmes.

\section{References}

1 Zur Hausen H. Viruses in human cancer. Science 1991; 254: 1167-1173.

2 Münger K, Scheffner M, Huibregtse JM, Howley PM. Interactions of HPV E6 and E7 with tumor suppressor gene products. Cancer Surv 1992; 12: 197-217.

3 Werness BA, Levine AJ, Howley PM. Association of human papillomavirus types 16 and 18 E6 proteins with p53. Science 1990; 248: 76-79.

4 Pei XF. The human papillomavirus E6/E7 genes induce discordant changes in the expression of cell growth regulatory proteins. Carcinogenesis 1996; 17: 1395-1401.

5 Jones DL, Münger K. Interactions of human papillomavirus E7 protein with cell cycle regulators. Semin Cancer Biol 1996; 7: 327-337.

6 Gulliver GA, Herber RL, Liem A, Lambert PF. Both conserved region 1 (CR1) and CR2 of the human papillomavirus type 16 E7 oncogene are required for induction of epidermal hyperplasia and tumor formation in transgenic mice. J Virol 1997; 71: 5905-5914.

7 Porreco $\mathrm{R}$ et al. Gynecological malignancies in immunosuppressed organ homograft recipients. Obstet Gynecol 1975; 45: 359-364.

8 Johnson JC et al. High frequency of latent and clinical human papillomavirus cervical infections in immunocomprised human immunodeficiency virus-infected women. Obstet Gynecol 1992; 79: 321-327.

9 Feltkamp MC et al. Vaccination with cytotoxic T lymphocyte epitope containing peptide protects against a tumor induced by human papillomavirus type 16-transformed cells. Eur J Immunol 1993; 23: 2242-2249.

10 Feltkamp MC et al. Cytotoxic T lymphocytes raised against a subdominant epitope offered as a synthetic peptide eradicate human papillomavirus type 16-induced tumors. Eur J Immunol 1995; 25: 2638-2642.

11 Jochmus I et al. Specificity of human cytotoxic T lymphocytes induced by a human papillomavirus type 16 E7-derived peptide. J Gen Virol 1997; 78: 1689-1695.

12 De Bruijn ML et al. Immunization with human papillomavirus type 16 (HPV16) oncoprotein-loaded dendritic cells as well as prrotein in adjuvant induces MHC class I-restricted protection to HPV16-induced tumor cells. Cancer Res 1998; 58: 724-731.

13 Strauss JH, Strauss EG. The alphaviruses: gene expression, replication, and evolution. Microbiol Rev 1994; 58: 491-562.

14 Liljeström P, Lusa S, Huylebroeck D, Garoff H. In vitro mutagenesis of a full-length cDNA clone of Semliki Forest virus: the small 6,000-molecular-weight membrane protein modulates virus release. J Virol 1991; 65: 4107-4113. 
15 Liljeström P, Garoff H. A new generation of animal cell expression vectors based on the Semliki Forest virus replicon. Biotechnol 1991; 9: 1356-1361.

16 Berglund $\mathrm{P}$ et al. Semliki Forest virus expression system: production of conditionally infectious recombinant particles. Biotechnol 1993; 11: 916-920.

17 Sedman SA et al. The full-length E6 protein of human papillomavirus type 16 has transforming and trans-activating activities and cooperates with E7 to immortalize keratinocytes in culture. J Virol 1991; 65: 4860-4866.

18 Greenfield I, Nickerson J, Penman S, Stanley M. Human papillomavirus 16 E7 protein is associated with the nuclear matrix. Proc Natl Acad Sci USA 1991; 88: 11217-11221.

19 Nindl I et al. The E7 protein of human papillomavirus (HPV) type 16 expressed by recombinant vaccinia virus can be used for detection of antibodies in sera from cervical cancer patients. J Virol Meth 1996; 62: 81-85.

20 Braspenning $\mathrm{J}$ et al. A general purification protocol for E7 proteins from 'high- and low-risk' human papillomavirus types expressed in the yeast Schizosaccharomyces pombe. Protein Expression Purif 1997; 10: 192-201.

21 Grossman SR, Mora R, Laimins LA. Intracellular localization and DNA-binding properties of human papillomavirus type 18 E6 protein expressed with a baculovirus vector. J Virol 1989; 63: 366-374.

22 Daniels PR, Sanders CM, Maitland NY. Characterization of the interactions of human papillomavirus type 16 E6 with p53 and E6-associated protein in insect and human cells. J Gen Virol 1998; 79: 489-499.

23 Toes REM et al. Peptide vaccination can lead to enhanced tumor growth through specific T-cell tolerance induction. Proc Natl Acad Sci USA 1996; 93: 7855-7860.

24 Boursnell MEG et al. Construction and characterization of a recombinant vaccinia expressing human papillomavirus proteins for immunotherapy of cervical cancer. Vaccine 1996; 14: 1485-1494.

25 Lin KY et al. Treatment of established tumors with a novel vaccine that enhances major histocompatibility class II presentation of tumor antigen. Cancer Res 1996; 56: 21-26.

26 Chen $\mathrm{L}$ et al. Induction of cytotoxic $\mathrm{T}$ lymphocytes specific for a syngeneic tumor expressing the E6 oncoprotein of human papillomavirus type 16. J Immunol 1992; 148: 2617-2621.

27 Borysiewicz LK et al. A recombinant vaccinia virus encoding human papillomavirus types 16 and 18, E6 and E7 proteins as immunotherapy for cervical cancer. Lancet 1996; 347: 1523-1527.

28 Glasgow GM, McGee MM, Sheahan BJ, Atkins GJ. Death mechanisms in cultured cells infected by Semliki Forest virus. J Gen Virol 1997; 78: 1559-1563.

29 Berglund P, Fleeton MN, Smerdou C, Liljeström P. Immunization with recombinant Semliki Forest virus induces protection against influenza challenge in mice. Vaccine 1999; 17: 497507.

30 Rock KL. A new foreign policy: MHC class I molecules monitor the outside world. Immunol Today 1996; 17: 131-137.

31 Bennett SRM. Help for cytotoxic-T-cell responses is mediated by CD40 signalling. Nature 1998; 393: 478-480.

32 Lanzavecchia A. Immunology. Licence to kill. Nature 1998; 393: 413-414.

33 Ridge JP, Di Rosa F, Matzinger P. A conditioned dendritic cell can be a temporal bridge between a CD4+ T-helper and a Tkiller cell. Nature 1998; 393: 474-478.

34 Schoenberger SP et al. T-cell help for cytotoxic T lymphocytes is mediated by CD40-CD40L interactions. Nature 1998; 393: 480-483.

35 Zhou $\mathrm{X}$ et al. Generation of cytotoxic and humoral immune responses by nonreplicative recombinant Semliki Forest virus. Proc Natl Acad Sci USA 1995; 92: 3009-3013.

36 Berglund $\mathrm{P}$ et al. Outcome of immunization of Cynomolgus monkeys with recombinant Semliki Forest virus encoding human immunodeficiency virus type I envelope protein and challenge with a high dose of SHIV-4 virus. AIDS Res Hum Retroviruses 1997; 13: 1487-1495.

37 Smerdou C, Liljeström P. Two-helper RNA system for production of recombinant Semliki Forest virus particles. J Virol 1999; 73: 1092-1098.

38 Smits PHM, Smits HL, Jebbink MF, Ter Schegget J. The short arm of chromosome 11 likely is involved in the regulation of the human papillomavirus type 16 early enhancer-promoter and in the suppression of the transforming activity of the viral DNA. Virol 1990; 176: 158-165. 